\title{
Transaktionskosten bei Energieeffizienz-Investitionen in Unternehmen
}

\author{
Eine empirische Untersuchung in Energieeffizienz-Netzwerken Deutschlands
}

\author{
Michael Mai · Thorsten Gebhardt · Fabian Wahl · Julius Dann · Eberhard Jochem
}

Online publiziert: 28. November 2014

(C) Springer Fachmedien Wiesbaden 2014

\begin{abstract}
Zusammenfassung Hohe Rentabilitäten von Energieeffizienz-Investitionen mit durchschnittlich $30 \%$ interner Verzinsung werden immer wieder aus dem Kreis beratender Ingenieure und Technologieherstellern berichtet. Aber zugleich beklagen sie ihre mangelnde Umsetzung, selbst durch Spezialisten wie Contractoren. Die Mikro-Ökonomen erklären den Umsetzungsmangel $u$. a. durch hohe Transaktionskosten. Allerdings sind diese empirisch kaum erhoben, so dass ihre Bedeutung im Rahmen der gehemmten Energieeffizienz-Potentiale nicht konkret eingeschätzt werden kann. Der Artikel berichtet über 40 differenziert analysierte Transaktionskosten von verschiedenen Energieeffizienz-Investitionen, es ergibt sich eine klare Degression in Abhängigkeit von der Höhe der Investition und ersten Hinweisen zu weiteren Einflussfaktoren (z. B. der Komplexität der Investition und der Größe und Energieintensität des Unternehmens). Da die erhobenen Zahlen von teilnehmenden Unternehmen in Energieeffizienz-Netzwerken stammen und auch der Einfluss der Netzwerke und des Erfahrungsaustausches abgefragt wurde, gibt es erste Hinweise, welche der Transaktionskosten durch dieses Instrument am meisten reduziert werden könnten.
\end{abstract}

\section{Transaction costs of energy efficiency investments in companies}

Summary Consulting engineers and technology suppliers keep reporting on high profitability figures of internal rate

E. Jochem $(\varangle)$

Fraunhofer ISI, Breslauer Str. 48, Karlsruhe, Deutschland

E-Mail: Eberhard.Jochem@isi.fraunhofer.de

M. Mai · T. Gebhardt · F. Wahl · J. Dann

IREES, Schönfeld Str. 8, Karlsruhe, Deutschland of return at an average of $30 \%$. However, they simultaneously complain about lack of implementation, even by specialists like contractors. The micro economists explain this low rate of implementation besides others by high transaction costs. However, there are almost no empirical data available. This means that their influence as obstacle for implementing profitable energy efficiency investments cannot be assessed. The article reports on 40 thoroughly analysed energy efficiency investments and their transaction costs. There is an obvious decline of the transaction costs with increasing investments; first hints to further influences could be identified such as the complexity of the investment, the size and the energy intensity of the company. As the empirical data stem from companies participating in so called energy efficiency networks and as the impact of the exchange of experience in those networks was analysed, the findings also classified those parts of the transaction costs that energy efficiency networks are likely to reduce most.

\section{Transaktionskosten als Hemmnis für Investitionen in Energieeffizienz in Unternehmen}

Das jährliche Einsparpotential durch einen effizienteren Umgang mit Energie beträgt in der deutschen Industrie derzeit rund 10 Mrd. $€$ (vgl. BMU 2010). Steigende Energieträgerpreise würden diese Beträge ansteigen lassen. Allerdings werden in Unternehmen die Einsparpotentiale trotz ihrer Wirtschaftlichkeit oft nur zögerlich in der Praxis umgesetzt (vgl. Seefeldt et al. 2007; Schmid et al. 2003; Schröter et al. 2009; Jochem et al. 2010). Diese Umsetzungslücke wird auf verschiedene unternehmensinterne und -externe Hemmnisse für Energieeffizienz-Maßnahmen zurückgeführt (vgl. Jaffe und Stavins 1994; Levine et al. 
1994), in jüngerer Zeit auch auf ungenutzte fördernde Faktoren (Jochem et al. 2014). Eines dieser Hemmnisse stellen hohe Transaktionskosten (TAK) bzw. der fehlende Marktüberblick bei den Verantwortlichen dar vgl. Jochem et al. 2010; Seefeldt et al. 2007; Schmid et al. 2003; Jaffe und Stavins 1994; Hein und Blok 1995. Unter Transaktionskosten werden dabei allgemein die Ressourcen verstanden, die durch den Austausch von Gütern und Leistungen aufgewendet werden müssen. Hierunter fallen Such- und Informationskosten, Verhandlungs- und Entscheidungskosten, Überwachungs- und Durchsetzungskosten (vgl. Richter et al. 2003, S. 40-41).

Einen Ansatz, das Hemmnis hoher Transaktionskosten $\mathrm{zu}$ reduzieren, bieten Energieeffizienz-Netzwerke. Hierunter versteht man den Zusammenschluss von 10-15 Industrieunternehmen aus unterschiedlichen Branchen in einer bestimmten Region. Die teilnehmenden Unternehmen setzen es sich u. a. zum Ziel, durch einen moderierten Erfahrungsaustausch im Netzwerk die Transaktionskosten $\mathrm{zu}$ reduzieren und so ihre Energieeffizienz schneller zu steigern als wenn jedes Unternehmen für sich allein bestehende Energieeffizienz-Investitionspotentiale realisieren würde (vgl. Jochem et al. 2010). Derzeit laufende Analysen versuchen, das Hemmnis hoher Transaktionskosten bei Energieeffizienz-Investitionen in Unternehmen, die an solchen Energieeffizienz-Netzwerken teilnehmen, genauer zu untersuchen.

Dazu wird in Abschn. 2 zunächst das Konzept der Transaktionskosten aus der Literatur beschrieben und die Bedeutung der Transaktionskosten bei EnergieeffizienzMaßnahmen erläutert. Abschnitt 3 beschreibt das Forschungsdesign. In Abschn. 4 werden die Ergebnisse der Studie dargestellt und in Abschn. 5 werden diese Ergebnisse diskutiert. Abschließend wird in Abschn. 6 die Arbeit zusammengefasst und es werden Empfehlungen für den weiteren Untersuchungsverlauf gemacht.

\section{Transaktionskosten bei Energieeffizienz-Maßnahmen}

Transaktionen sind kostspielig und werden häufig von den Unternehmen nicht bewusst wahrgenommen. Mit dieser wichtigen Erkenntnis versucht die Transaktionskostentheorie die Eigenschaften von realen Märkten in ihren Ansätzen zu berücksichtigen (vgl. Osterheld 2000, S. 90-92; Richter et al. 2003, S. 53). Anders als in den idealisierten Annahmen der neoklassischen Modelle wird die Vorstellung der vollständigen Markttransparenz und dem kostenlosen Austausch von Gütern aufgehoben. Es ist den Marktteilnehmern nicht mehr möglich, sich vollständig und kostenlos zu informieren. Jeder Nachfrager nach einer Investition wird vor das Problem gestellt, dass er Zeit und Mühe darauf verwenden muss, um Informationen über diese beabsichtigte Investition zu beschaffen. Die Güter und Leistungen sind aus Sicht der Marktteilnehmer nicht homogen, denn es gibt räumliche, zeitliche und sachliche Präferenzen. Die Produkte sind damit spezifisch und nicht mehr einfach und kostenlos durch andere ersetzbar (vgl. Göbel 2002, S. 3). Diese Kosten, die durch den Austausch von solchen Gütern und Leistungen aufgewendet werden müssen, heißen Transaktionskosten (vgl. Richter et al. 2003, S. 40-41).

\subsection{Allgemeine Definition von Transaktionskosten}

Hinsichtlich einer allgemeinen Definition von Transaktionskosten besteht in der Literatur keine Einigkeit. Eine sehr gebräuchliche Definition ist die von Williamson (1990, S. 1): „Eine Transaktion findet statt, wenn ein Gut oder eine Leistung über eine technisch trennbare Schnittstelle hinweg übertragen wird“. Die Transaktionskosten sind die Summe aller Kosten der Aktivität Transaktion. Eine etwas anschaulichere Definition schlagen Richter et al. (vgl. 2003, S. 40-41) vor: Transaktionskosten sind allgemein die Ressourcen, die durch den Austausch von Gütern und Leistungen aufgewendet werden müssen. Hierunter fallen Such- und Informationskosten, Verhandlungsund Entscheidungskosten aber auch Überwachungs- und Durchsetzungskosten.

In der vorliegenden Arbeit werden unter den Transaktionskosten einer Energieeffizienz-Maßnahme alle Kosten verstanden, die im Zusammenhang mit Informations-, Entscheidungs- und Kommunikationsvorgängen bei der Anbahnung, Vereinbarung, Kontrolle und Anpassung einer solchen Investition anfallen. Aus Sicht des investierenden Unternehmens sind dafür Aktivitäten bzw. Tätigkeiten erforderlich, die von den Mitarbeitern des Unternehmens ausgeführt werden. Diese betriebsinternen, zeitlichen Aufwendungen machen den einen Teil der Transaktionskosten aus, die bei der Umsetzung einer EnergieeffizienzMaßnahme anfallen. Hinzu kommen Kosten, die dadurch entstehen, dass die Ausführung dieser Tätigkeiten unterstützt bzw. übernommen wird, wie beispielsweise externe Berater-Honorare.

2.2 Relevanz von Transaktionskosten bei EnergieeffizienzInvestitionen

Bereits Coase (1960, S.15) erkannte, dass die Transaktionskosten so hoch sein können, dass Transaktionen dadurch vollständig verhindert werden. In diesem Zusammenhang spricht man - oft auch sehr vereinfachend und summarisch - von Marktversagen, das immer dann auftrete, wenn die Prämissen der neoklassischen Modelle verletzt werden (vgl. Göbel 2002, S. 30, 155). Gerade im Zusammenhang mit Energieeffizienz-Investitionen wird dabei häufig die 
Rentabilität der Maßnahmen mit der Begründung bestritten, dass bei der Wirtschaftlichkeitsberechnung die Transaktionskosten nicht berücksichtigt werden (Hein und Blok 1995; Grubb et al. 1993). Als kostentreibende Einflussfaktoren werden dabei hauptsächlich die Such- und Entscheidungskosten genannt, denn die Kosten für Entscheidungen zu Energieeffizienz-Maßnahmen werden in den meisten Unternehmen nur selten bewusst reflektiert (vgl. Jochem et al. 2010).

Diese Kosten werden in den Unternehmen oft nicht einmal im Ansatz quantifiziert. Darauf hingewiesen antworteten viele Geschäftsführer bei den Erhebungen der Transaktionskosten, dass diese zu den Gemeinkosten zählen oder dass sie bei qualitativer Abwägung gegenüber anderen Kosten (z. B. für Gewerbeaufsichtsmaßnahmen oder Sicherheit) zu hoch seien oder in den allgemeinen Tätigkeiten für Energie, Umwelt und Betriebssicherheit keine große Rollen spielen. Die Honorarordnung für Architekten und Ingenieure (HOAI 2013, § 53-56) unterscheidet zwar auch nach acht Leistungsphasen - von der Grundlagenerhebung bis zur Bau- bzw. Objektüberwachung -, aber diese sind aus der Sicht der Leistungen eines beratenden Ingenieurs beschrieben und nicht aus der Sicht der erforderlichen Arbeiten des Unternehmens.

\section{Erhebung der Transaktionskosten bei Energieeffizienz-Maßnahmen in Unternehmen}

Zur Erhebung von empirischen Daten für Transaktionskosten von Energieeffizienz-Investitionen wurden meist mittelständische Unternehmen aus dem Forschungsprojekt 30 Pilotnetzwerke ${ }^{1}$ befragt. Ziel des Projekts war der Aufbau und die Betreuung von 30 Energieeffizienz-Netzwerken in Deutschland. Dabei wurden neu entstehende Netzwerke bezuschusst und deren Arbeit laufend wissenschaftlich begleitet. Zielgruppe des Projekts sind mittlere Betriebe bzw. Unternehmen aus dem Industriesektor (vgl. Fraunhofer-Institut für System- und Innovationsforschung ISI 2014).

\subsection{Forschungsdesign}

Die Befragung erfolgte über einen Fragebogen, der an die Unternehmen versendet wurde. Ziel dieses Fragebogens war, Informationen zu den Transaktionskosten einer konkreten und abgeschlossenen, investiven EnergieeffizienzMaßnahme zu erfassen (vgl. Gebhardt 2011; Wahl 2012; Dann 2013). In Anlehnung an Hein und Blok (1995) wurde

\footnotetext{
${ }^{1} \mathrm{Zu}$ weiteren Informationen über das Projekt 30 Pilotnetzwerke sei an dieser Stelle auf die offizielle Projekt-Website verwiesen: http://30pilot-netzwerke.de/nw-de/.
}

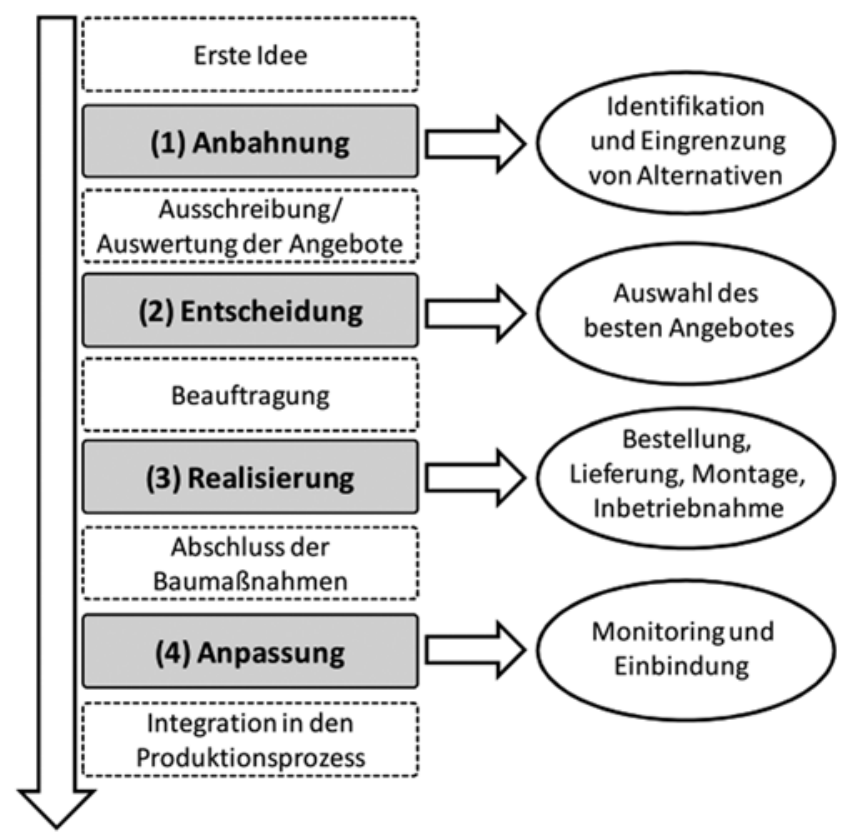

Abb. 1 Transaktionskosten nach Ablaufphasen von Investitionen

für die Befragung ein Investitionsmodell genutzt, welches den idealtypischen Ablauf einer Energieeffizienz-Investition in vier Phasen einteilt: 1) Anbahnungsphase, 2) Entscheidungsphase, 3) Realisierungsphase und 4) Anpassungsphase (siehe Abb. 1). Diese Struktur wurde im Rahmen der Studie durch die Unternehmen bestätigt. Für jede der Phasen wurden die beteiligten Mitarbeiter, die zeitlichen Aufwendungen für transaktionskostenrelevante Tätigkeiten und die Kosten für externe Beratungsleistungen erfasst.

Um die Transaktionskosten zu ermitteln, wird die Dauer der zeitlichen Aufwendungen der beteiligten Personen mit einem Stundensatz in Höhe von $60 €$ multipliziert, der die durchschnittlichen Kosten für eine Arbeitsstunde beinhaltet. Als zweiter Bestandteil der Transaktionskosten werden die Kosten für externe Beratungsleistungen hinzugerechnet.

Als Transaktionskosten bezüglich einer EnergieeffizienzInvestition wurden in der Studie nur die Transaktionskosten untersucht, die beim Investor anfallen. Bei Contractoren sind diese Transaktionskosten u. a. eingepreist. Bei sehr geringen Energieeffizienz-Investitionssummen sind diese Transaktionskosten einschließlich der Verhandlungskosten mit dem Contracting-Nehmer so hoch, dass diese Investitionen vom Contractor gar nicht angeboten wird. Beim Verkäufer bzw. beim Zulieferer entstehen ebenfalls Transaktionskosten. Diese sollten jedoch im Verkaufspreis enthalten sein und werden daher im Rahmen der vorliegenden Arbeit nicht weiter behandelt (vgl. Hein und Blok 1995; Eikmeier et al. 2009). 
Abb. 2 Deskriptive Statistik der erhobenen 40 Investitionen zur Erhebung der Transaktionskosten
Statistiken der Unternehmen

\begin{tabular}{|lc|}
\hline Branche & Anzahl der Unternehmen \\
Kunststoffverarbeitung & 7 \\
Metall- und Holzverarbeitung & 6 \\
Nahrungs- u. Genussmittel & 5 \\
Automobilindustrie (allg.) & 3 \\
Dienstleistungen & 3 \\
Baugewerbe/Bau & 3 \\
Sonstige & 8 \\
Summe & 35 \\
\hline
\end{tabular}

\begin{tabular}{|lc|}
\hline Anzahl der Mitarbeiter & Anzahl der Unternehmen \\
$>250$ & 25 \\
$<=250$ & 7 \\
$<50$ & 2 \\
Keine Angabe & 1 \\
Summe & 35 \\
\hline
\end{tabular}

Energieintensität

Max. $\quad 1.000 .000 \mathrm{MWh} / \mathrm{a}$

Min.

Mittelwert

Standardabweichung

Median

Keine Angabe

Statistiken der Maßnahmen

\begin{tabular}{|lc|}
\hline Art der Maßnahme & Anzahl der Maßnahmen \\
Neuinvestition & 8 \\
Ersatz- und Rationalisierungsinvestition & 31 \\
Keine Angabe & 1 \\
Summe & 40 \\
\hline
\end{tabular}

\begin{tabular}{|lr|}
\hline Investitionssumme & \\
Max. & $800.000 €$ \\
Min. & $1.715 €$ \\
Mittelwert & $110.948 €$ \\
Standardabweichung & $170.301 €$ \\
Median & $50.000 €$ \\
Keine Angabe & 1 Maßnahme \\
\hline
\end{tabular}

\begin{tabular}{|lc|}
\hline Technologie & Anzahl der Maßnahmen \\
Isolierung/Wärmedämmung & 8 \\
Wärmerückgewinnung & 8 \\
Austausch Motoren/Pumpen & 3 \\
Kältetechnik & 3 \\
Wechsel Energieträger & 3 \\
Beleuchtung & 2 \\
Leckageverluste (Druckluft) & 2 \\
Raumlufttechnik & 2 \\
Sonstige & 8 \\
Keine Angabe & 1 \\
Summe & 40 \\
\hline
\end{tabular}

Abb. 3 Transaktionskosten anteilig an der Investitionssumme (ohne Transaktionskosten)

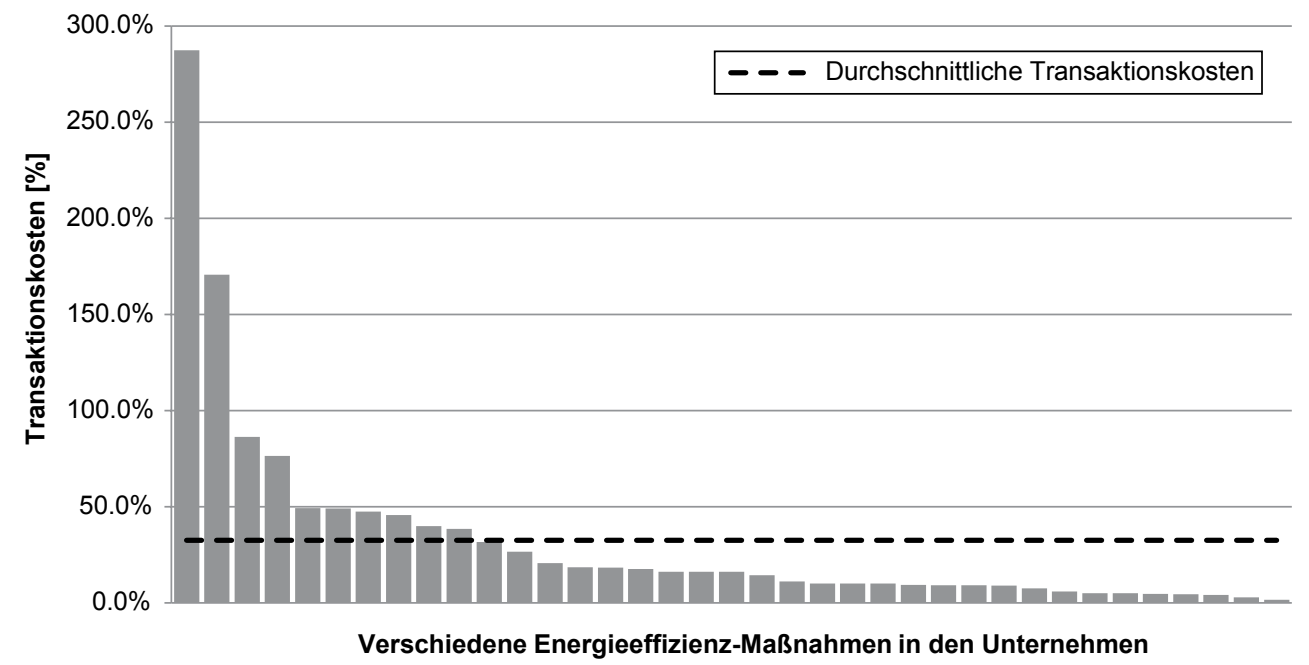

\subsection{Deskriptive Statistik}

Im Rahmen der Befragung wurden rund 100 Unternehmen angesprochen, von denen 35 Unternehmen einer Teilnahme mit einer relativ aufwändigen Beantwortung des Fragebogens zustimmten. Insgesamt konnten Daten zu 40 verschiedenen Energieeffizienz-Investitionen erhoben werden. Enthalten sind Energieeffizienz-Maßnahmen unterschiedlichster Technologien mit Investitionssummen zwischen $1715 €$ und $800.000 €$ (vgl. Abb. 2). Die meisten Investitionen sind Ersatz- und Rationalisierungsmaßnahmen. Die betrachteten Industrieunternehmen kommen aus unterschiedlichen Branchen (Schwerpunkt GebrauchsgüterIndustrie) und haben Produktionsstandorte mit 25 bis $\mathrm{zu}$ 3000 Mitarbeitern. Der Jahresenergieverbrauch der befragten Unternehmen (eines Produktionsstandortes) liegt zwischen 1,3 bis $1000 \mathrm{GWh} / \mathrm{a}$.

\section{Ergebnisse}

Die ermittelten Transaktionskosten für die einzelnen Energieeffizienz-Investitionen in den befragten Unternehmen zeichnen sich durch eine extrem große Spannweite aus und 
Tab. 1 Transaktionskosten nach Ablaufphasen, anteilig an den gesamten Transaktionskosten (arithmetische Werte)

\begin{tabular}{ll}
\hline Anbahnungsphase & $41 \%$ \\
Entscheidungsphase & $26 \%$ \\
Realisierungsphase & $24 \%$ \\
Anpassungsphase & $9 \%$ \\
\hline
\end{tabular}

betragen zwischen 1,7 und $287 \%$ der Investitionssumme (ohne Transaktionskosten; vgl. Abb. 3). In zwei Fällen übersteigen die Transaktionskosten die eigentliche Investitionssumme, die in beiden Fällen relativ gering ist $(<10.000 €)$. Die durchschnittlichen Transaktionskosten liegen bei $33 \%$ der Investitionssumme, mit einer Standardabweichung von $\mathrm{s}=54 \%$.

Die o. g. Transaktionskosten mit dem höchsten Anteil von $287 \%$ sind ein interessanter Fall. Die ursprünglich vom Hersteller angebotene Lösung hätte $300 \%$ der letztlich getätigten Investition ausgemacht (zzgl. Transaktionskosten). Da der Energieanwender diese Lösung für zu teuer hielt, recherchierte er mit hohem Such- und Reiseaufwand nach einer günstigeren Lösung, die er mit einem Drittel der ursprünglich angebotenen Lösung auch fand und investierte.

\subsection{Transaktionskosten nach Phasen}

Die Zerlegung der Transaktionskosten nach den vier Phasen (vgl. Abb. 1) kommt zu dem Ergebnis, dass die anfallenden Transaktionskosten mit laufendem Projektfortschritt abnehmen. Während die Anbahnungsphase im Mittel 41\% der gesamten Transaktionskosten ausmacht, fallen in der Anpassungsphase nur noch durchschnittlich $9 \%$ an (vgl. Tab. 1). Den größten Anteil der Transaktionskosten bei Energieeffizienz-Investitionen machen demnach mit zwei Dritteln die Such- und Entscheidungskosten vor der eigentlichen Umsetzung der Maßnahme aus. Im weiteren Projektverlauf (Realisierung und Anpassung) fallen im Durchschnitt dann insgesamt nur noch ein Drittel der gesamten Transaktionskosten an. Die Anpassungskosten zeigen dabei durchweg geringe Größenordnungen auf. In keinem der untersuchten
Fälle traten während der Anpassungsphase (Phase 4) mehr als ein Viertel an den gesamten Transaktionskosten auf.

\subsection{Einfluss von Transaktionskosten auf die Wirtschaftlichkeit von Energieeffizienz Maßnahmen}

Zur Berechnung des Risikos und der Wirtschaftlichkeit wurde für die untersuchten Maßnahmen zusätzlich die jährliche Kostenminderung („Gewinn“) erfasst, die sich durch den effizienteren Umgang mit den Energieträgern ergab. Zusammen mit den Kosten, die für eine Investition aufzuwenden sind, können daraus Amortisationszeit und interne Verzinsung der betroffenen Investition (mit und ohne Transaktionskosten) ermittelt werden.

Die Amortisationsmethode ermittelt über die Kapitalbindungsdauer einer Investition ihr Risiko (vgl. VDI 1996, S. 60). Hierfür wird der Zeitraum berechnet, in dem sich die Anschaffungskosten aus dem jährlichen Gewinnbeitrag der Investition refinanzieren (vgl. Ostertag et al. 2000, S. 62). Untersuchungen zeigen, dass Unternehmen allgemein eine Amortisationsdauer von zwei bis sechs Jahren von Investitionen fordern (vgl. Gruber et al. 1991; Hein und Blok 1995; Koot et al. 1984 zitiert nach Hein et al.) mit deutlicher Präferenz zwischen zwei und drei Jahren. Dies konnte auch im Rahmen dieser Erhebung bestätigt werden, wo der Durchschnitt der Amortisationszeit (ohne Transaktionskosten) bei zwei Jahren liegt (vgl. Abb. 4). In $85 \%$ der Fälle werden in Unternehmen lediglich die Ergebnisse zur Amortisationszeit als Entscheidungskriterium herangezogen (Schröter und Buschak 2009).

Ermittelt man die beiden Kennzahlen einmal ohne und einmal mit Transaktionskosten, so erhöht der Einbezug der Transaktionskosten die durchschnittliche Amortisationsdauer um etwa $25 \%$ und vermindert die interne Verzinsung im Durchschnitt um etwa 16\% für das untersuchte Sample der Energieeffizienz-Investitionen (vgl. Abb. 4).

Die untersuchten Fälle zeigen Amortisationszeiten von durchschnittlich 2,1 Jahren, mit einer Standardabweichung von $\mathrm{s}=1,5$ Jahren (siehe Abb. 4 links). Die Mehrzahl der
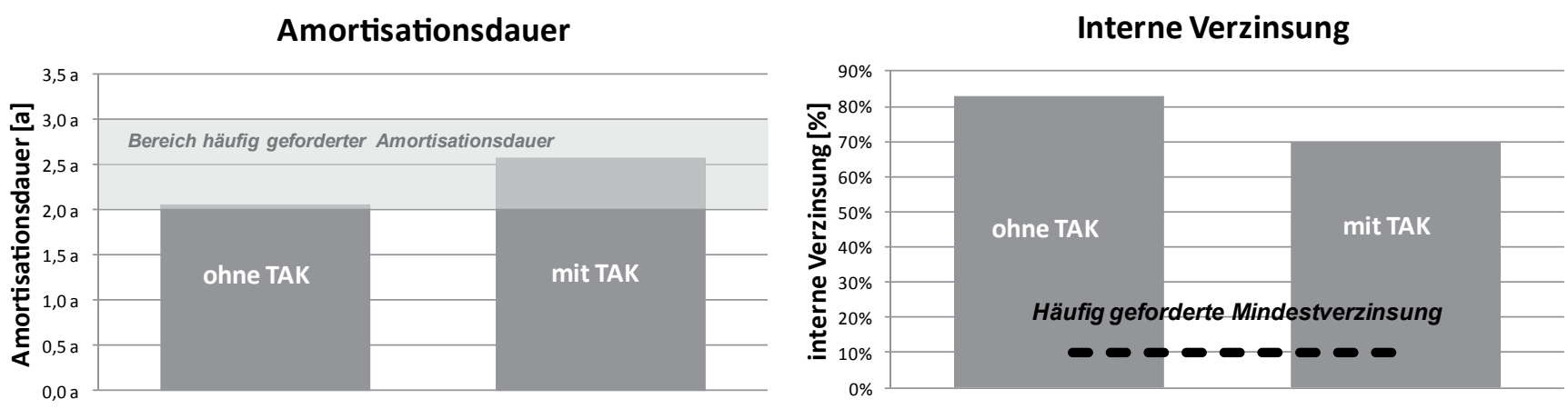

Abb. 4 Einfluss der Transaktionskosten (TAK) auf die Amortisationsdauer und die Wirtschaftlichkeit von verschiedenen EnergieeffizienzMaßnahmen $(n=40)$ 
umgesetzten Energieeffizienz-Maßnahmen (63\%) amortisierte sich dabei in weniger als drei Jahren. Werden die Transaktionskosten der Maßnahme berücksichtigt, also zur Investitionssumme addiert, steigt die durchschnittliche Amortisationsdauer um 0,5 Jahre auf 2,6 Jahre. Als Folge amortisieren sich noch 59\% der Maßnahmen innerhalb von drei Jahren. Damit können die Transaktionskosten durchaus ein Hemmnis bei der Investition in Energieeffizienz darstellen, wenn Unternehmen ihre Ansprüche an die Amortisationszeit an die dargestellte untere Grenze des abgebildeten Korridors von beispielsweise zwei Jahren anlegen und sich über die Rentabilität der Investition keine Rechenschaft geben.

Die alleinige Bewertung von Energieeffizienz-Investitionen mittels Amortisationszeit ist sehr kritisch zu bewerten. Zwar ist es richtig, dass je länger ein Kapitalbetrag gebunden ist, desto länger die Gefahr eines Verlustes besteht, aber bei den hier analysierten Investitionen im Bereich der Querschnittstechniken (Wärme-/Kälteerzeugung, Drucklufterzeugung, Lüftungsanlagen, Pumpen, Elektroantriebe oder Beleuchtung) kann man von einer Lebensdauer von 10 bis 20 Jahren ausgehen. In der Regel würde der Betrieb, der nur nach der Amortisationszeit entscheidet, in wenigen Jahren einen sehr energiekosten-intensiven Kapitalstock haben. Aus diesem Grund sollte die Amortisationszeitenrechnung bei Energieeffizienz-Maßnahmen nur als ergänzendes Kalkül verwendet werden, und das Rentabilitäts-Ergebnis von Barwert oder interner Verzinsung, bzw. Gesamtkapitalrendite als prioritäres Entscheidungskriterium (vgl. Ostertag et al. 2000, S. 62-64).

Ein selten genutztes Entscheidungskriterium ist die Berechnung des internen Zinssatzes. Er ermittelt die mittlere, jährliche Rendite einer Investition für ihre angenommene Nutzungsdauer. In der Wirtschaft sind Mindestverzinsungen von 7 bis $15 \%$ weit verbreitet (vgl. Erichsen 2011, S. 103). Die Höhe des Zinssatzes hängt dabei im Wesentlichen davon $a b$, welchen Risiken die Investition unterliegt. Da Investitionen in Energieeffizienz allgemein als risikoarm gelten (vgl. Ostertag et al. 2000, S. 62-64), wurde für diese Analyse eine Mindestverzinsung von 10\% angenommen.

Die meisten untersuchten Energieeffizienz-Maßnahmen zeigten deutlich höhere Verzinsungen als $10 \%$. Durchschnittlich lag die Verzinsung bei $83 \%$ (siehe Abb. 4 rechts). Insgesamt lagen 19\% der untersuchten Effizienz-Investitionen unter der angestrebten 10\%-Marke. Bei Berücksichtigung der Transaktionskosten sinkt die durchschnittliche interne Verzinsung auf $73 \%$. Als Folge weisen immer noch drei Viertel (zuvor $81 \%$ ) der Investitionen eine Mindestverzinsung von $10 \%$ auf. Die Transaktionskosten stellen in den untersuchten Fällen kein wesentliches Hemmnis dar, wenn sie den Durchschnitt der internen Verzinsung des untersuchten Sample um knapp 16\% reduzieren (vgl. Abb. 4, rechts).
Tab. 2 Statistische Analyse der Einflussfaktoren auf die Transaktionskosten $(n=40)$. (Quelle: eigene Berechnungen)

\begin{tabular}{lll}
\hline Unabhängige Variable & $\begin{array}{l}\text { Korrelationskoeffizient } \\
\text { nach Kendall-Tau-b }\end{array}$ & Signifikanz \\
\hline Investitionssumme & $-0,455$ & 0,000 \\
$\begin{array}{l}\text { Energieintensität (Gemes- } \\
\text { sen am Jahresenergiever- }\end{array}$ & $-0,306$ & 0,013 \\
brauch des Standorts) & & \\
\hline
\end{tabular}

Die Analyse ergab auch, dass Maßnahmen zur energetischen Optimierung der Gebäudehülle die üblich geforderten Amortisationszeiträume und Verzinsungen nicht erreichten, ebenso einige anlagentechnische Maßnahmen, teils Sonderlösungen wie der Einbau von Kondensationsblechen in Waschbädern oder der Ersatz von brennstoffbetriebenen Gabelstaplern durch Hybridstapler. In diesen Fällen wurden die gesamten, meist sehr hohen Investitionen der Energiekostenersparnis gegenübergestellt. In diesen und in Fällen von ohnehin erforderlichen Ersatzinvestitionen wurden von den Unternehmen oder energietechnischen Beratern die Methode des Differenzkostenansatzes nicht gewählt, mit dem Rentabilität und Risiko hätten bestimmt werden müssen.

\subsection{Einflussfaktoren auf die Höhe der Transaktionskosten in Unternehmen}

Als weitere Forschungsfrage wurden in der Analyse mögliche Einflussfaktoren untersucht, die die Höhe der Transaktionskosten mitbestimmen könnten. Dazu wurden die unabhängigen Variablen: Investitionssumme, Spezifität der Maßnahme, Häufigkeit ähnlicher Maßnahmen, Unternehmensgröße, Energieintensität am Standort und das Vorhandensein eines Energieverantwortlichen geprüft. Mittels statistischer Testverfahren wurde untersucht, ob diese Variablen eine Korrelation mit den anteiligen Transaktionskosten an der Investitionssumme aufweisen.

Die Tests ergaben, dass die Investitionssumme und die Energieintensität des Standorts mit den Transaktionskosten signifikant negativ korrelieren (vgl. Tab. 2). Dieses Ergebnis kann so interpretiert werden, dass mit zunehmender Energieintensität an einem Produktionsstandort die energietechnische Kompetenz zunimmt und damit die Such- und Entscheidungskosten für eine Einzel-Investition wegen des guten Vorwissens und eine guten Marktüberblicks sinken. Die untersuchten Energieeffizienz-Maßnahmen zeigten dabei in Unternehmen ab einem Jahresenergieverbrauch von etwa $20.000 \mathrm{MWh} / \mathrm{a}$ vergleichsweise niedrige Werte der Transaktionskostenanteile (meist unter 20\% der Investitionssumme).

Letztlich ist diese Abnahme des Transaktionskostenanteils mit zunehmender Investitionssumme auch deshalb zu erwarten, weil Such- und Entscheidungskosten relativ 
Abb. 5 Regression der Transaktionskosten über der Investitionssumme $(n=40)$

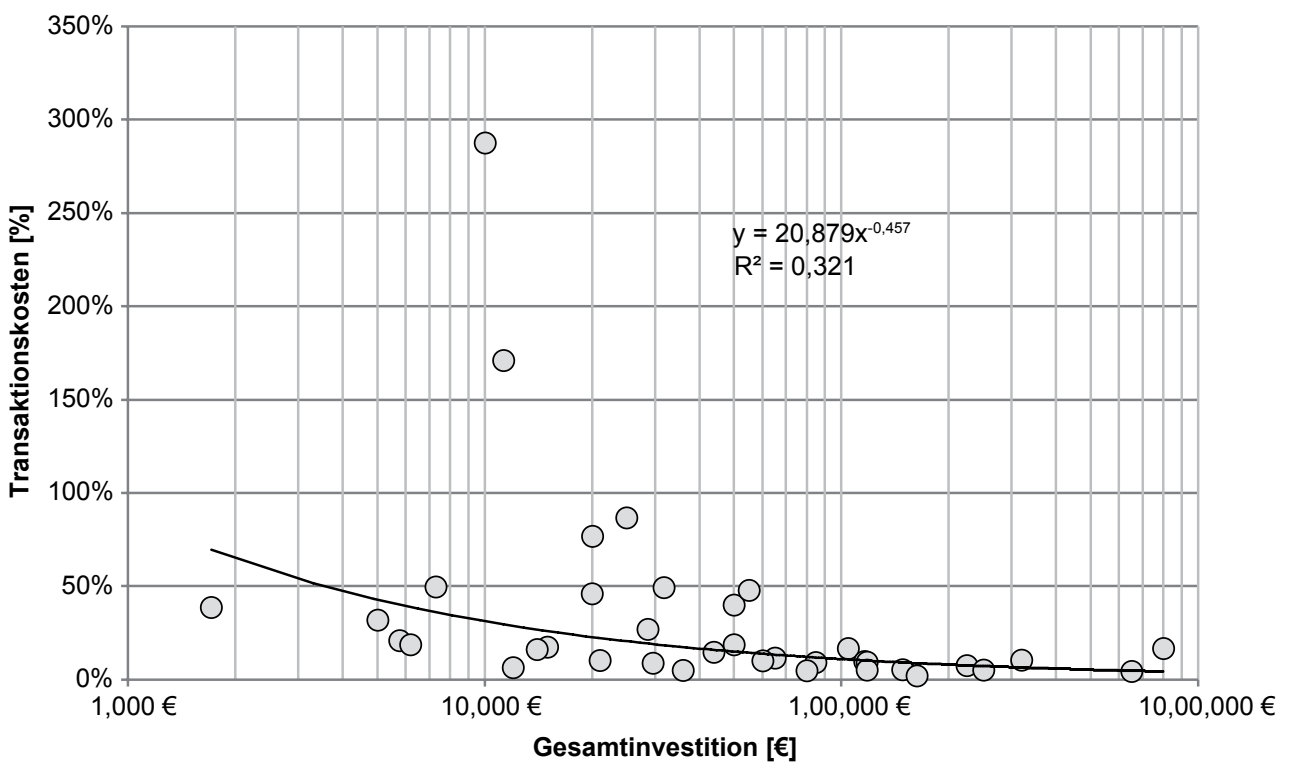

gleich hoch sind, unabhängig von der Investitionshöhe (vgl. Bleyl und Seefeld 2012; Eikmeier et al. 2009).

Allerdings dürfte hier auch ein Zusammenhang zwischen Energieintensität am Standort und die Höhe der Investitionssumme vorliegen. Denn letztere korreliert ebenfalls signifikant negativ mit den Transaktionskosten einer Energieeffizienz-Maßnahme. Dieser Zusammenhang ist auf dem 5\%-igen Niveau signifikant. Dies bedeutet, dass der Anteil der Transaktionskosten an der Gesamtinvestition mit steigender Investitionssumme abnimmt. In den untersuchten Fällen fielen die Transaktionskosten ab einer Investitionssumme von etwa $60.000 €$ vergleichsweise niedrig aus und lagen durchgehend unter $20 \%$, während die Transaktionskosten bei geringeren Investitionssummen deutlich stärker streuten (vgl. Abb. 5). In zwei Fällen überstiegen die Transaktionskosten sogar die eigentliche Investitionssumme.

Die Regression zeigt, dass die Transaktionskosten des Samples mit $y=20,879 x^{-0,457}$ abnehmen, wobei y für die Transaktionskosten in Prozent und $\mathrm{x}$ für die Investitionssumme in Euro steht. Das Bestimmtheitsmaß für dieses Ergebnis beträgt $R^{2}=0,321$ (siehe Abb. 5).

\subsection{Erfahrungsaustausch im Netzwerk}

Um zu untersuchen, ob sich die Transaktionskosten bei Energieeffizienz-Maßnahmen durch die Teilnahme an einem Energieeffizienz -Netzwerk senken lassen, wurden die teilnehmenden Unternehmen der Analyse befragt, wie diese den Erfahrungsaustausch im Netzwerk allgemein
Abb. 6 Wirkungen des Erfahrungsaustauschs in Energieeffizienz-Netzwerken auf die Transaktionskosten $(n=35$ bzw. 26). (Quelle: eigene Erhebungen)

\section{Lassen sich Transaktionskosten durch den Erfahrungsaustausch im Netzwerk reduzieren?}

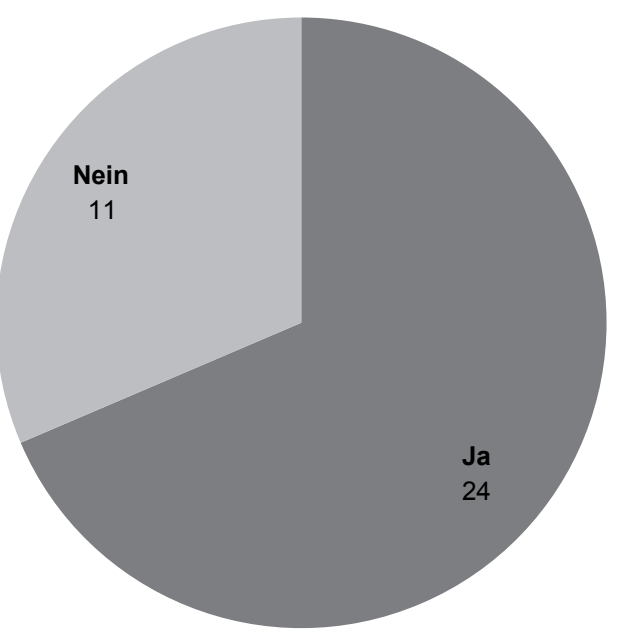

Konnten Sie bereits konkrete Einsparungen bei Ihren Arbeiten feststellen?

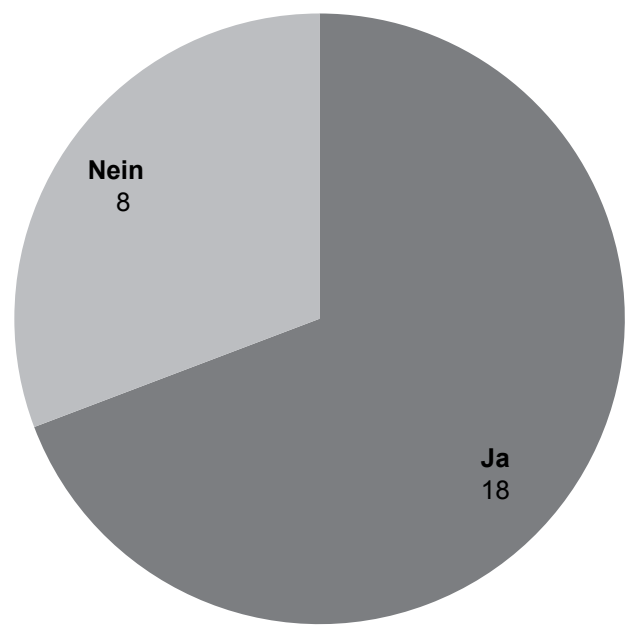


Abb. 7 Eingesparte transaktionskostenrelevante Tätigkeiten aufgrund des Erfahrungsaustauschs und bi-lateraler Kontakte in Energieeffizienz-Netzwerken $(n=40)$. (Quelle: eigene Erhebungen)

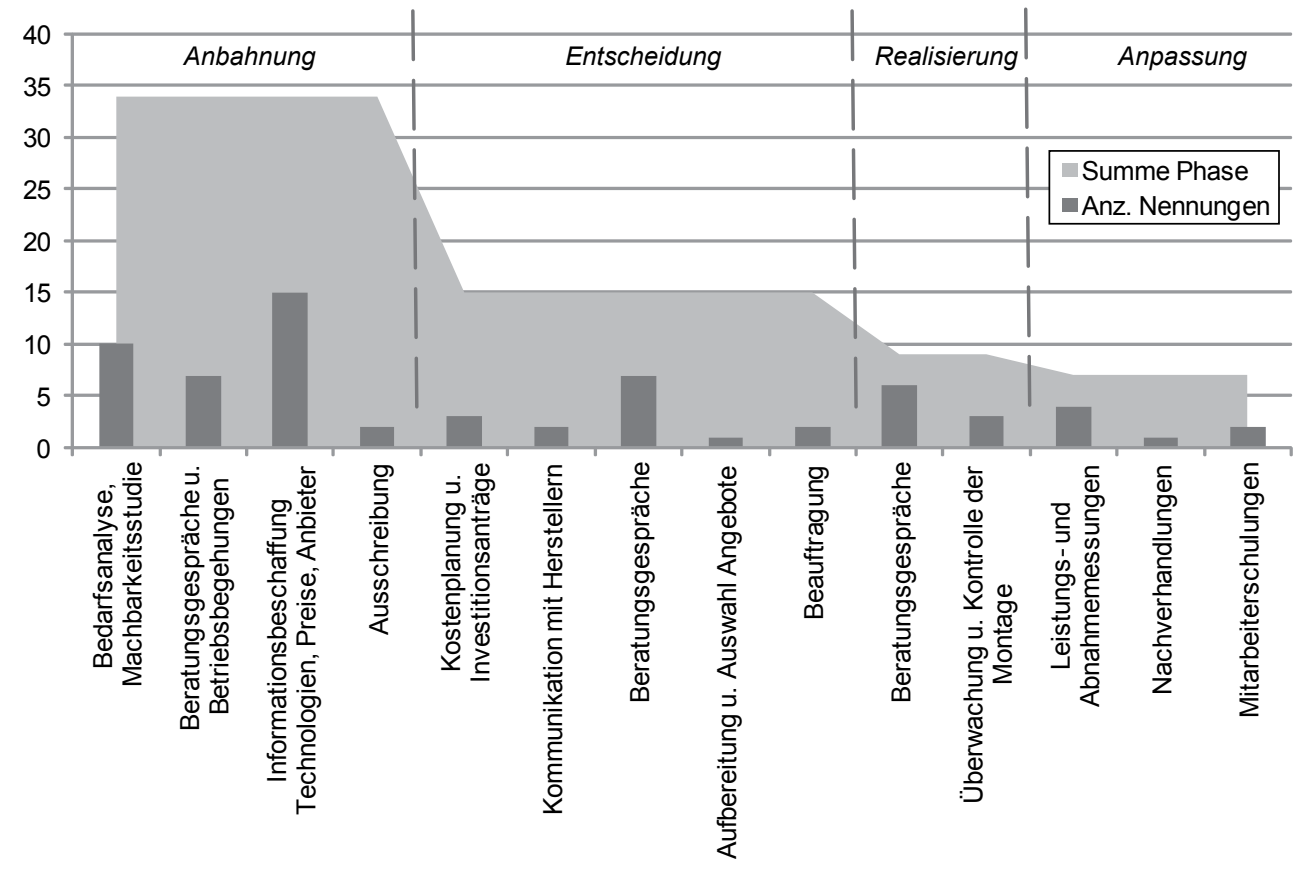

wahrnehmen. Auf die Frage hin, ob sich durch den Erfahrungsaustausch im Netzwerk potentiell Transaktionskosten senken lassen, antworteten 24 Unternehmen mit ,ja“ und 11 Unternehmen mit ,nein“. Weiterhin gaben 18 Unternehmen an, bereits Einsparungen bei den Transaktionskosten durch die Netzwerkarbeit im Unternehmen festgestellt zu haben (siehe Abb. 6).

Die Unternehmen wurden außerdem gebeten, solche Tätigkeiten zu nennen, bei denen bereits Einsparungen festgestellt werden konnten (Mehrfachnennungen waren möglich). Die Probanden gaben dabei meist Tätigkeiten in der Anbahnungs- und Entscheidungsphase an. Besonders die Informationsbeschaffung zu Technologien, Preisen und Anbietern wurde dabei überdurchschnittlich oft genannt (vgl. Abb. 7). Diese Antworten zeigen, dass ein Erfahrungsaustausch in den Netzwerken stattfindet und dadurch Transaktionskosten reduziert werden können. Über die Höhe dieser Einsparungen an den Transaktionskosten konnten die Befragten jedoch keine Aussage machen.

\section{Diskussion}

\subsection{Vergleich mit anderen Arbeiten}

Obwohl die Literatur zur Transaktionskostentheorie mittlerweile recht umfangreich ist, finden sich darin bisher nur wenige empirische Untersuchungen zur Höhe der Transaktionskosten bei Energieeffizienz-Investitionen.

Dazu gehört eine Fallstudie von Köwener und Schleich (2000). Die Autoren untersuchen die Kosten für Vertragsverhandlungen bei langfristigen Energiedienstleistungsverträ- gen in vier Unternehmen. In drei Fällen wurde die Frage mit einer Schätzung von 5-10\% an der gesamten Investitionssumme beantwortet. Eine explizite Erfassung der Transaktionskosten wird in der Erhebung nicht vorgenommen.

Ostertag (2003, S. 195-196, 224-228) erhebt in einer Fallstudie die Transaktionskosten bei der Beschaffung von (Hochleistungs-) Elektromotoren unterschiedlicher Motorleistungen in einem Großkonzern aus der Chemieindustrie. Sie beziffert die Transaktionskosten auf $300 €$ (für $1 \mathrm{~kW}$ ), $300 €$ (für $10 \mathrm{~kW}$ ) und $600 €$ (für $100 \mathrm{~kW}$ ). Ostertag kommt zu dem Schluss, dass für kleine Motorleistungen $(1 \mathrm{~kW})$ die Transaktionskosten damit den eigentlichen Anschaffungspreis von $200 €$ übersteigen. Eikmeier et al. 2009 kommen zu sehr ähnlichen Ergebnissen im Rahmen des Contracting im Mietwohnungsbau.

Hein und Blok (1995) liefern eine erste konkrete Quantifizierung von Transaktionskosten bei Energieeffizienz-Investitionen. In ihrer Studie ermitteln sie die Transaktionskosten in 12 energieintensiven Großunternehmen in den Niederlanden aus der Metall-, Chemie-, Papier-, Nahrungsmittel und Textilindustrie. Sie nutzen ebenfalls ein Investitionsmodell, welches vier verschiedene Phasen einer Investition differenziert. Diese Einteilung ermöglicht einen Vergleich der beiden Arbeiten.

Bei einem Vergleich mit den Ergebnissen von Hein und Blok (1995) zeigen die Resultate der vorliegenden Studie vordergründig deutliche Abweichungen auf. Hein und Blok ermitteln für die Transaktionskosten nur 3-8\% der Investitionssumme. Die Autoren beziehen sich in ihrer Untersuchung allerdings auf Maßnahmen mit Investitionssummen zwischen 1 Mio. $€$ und 13 Mio. $€$. In der vorliegenden Arbeit gibt es nur eine Maßnahme, die mit $800.000 €$ ähn- 
liche Größenordnungen erreicht. Die Gleichung in Abb. 5 bestätigt aber die Transaktionskosten-Anteile von Hein und Blok (1995), wenn man die hohen Investitionssummen in die Gleichung einsetzt. Ein geringer Unterschied mag auch dadurch entstehen, dass Hein und Blok (1995) keine Transaktionskosten während der Implementierung erfassen, da nach ihrer Auffassung diese Phase nicht transaktionskostenrelevant ist. In der vorliegenden Arbeit fallen aber während der Realisierung von Energieeffizienz-Investitionen Transaktionskosten in Form von Überwachungs- und Durchsetzungskosten an, wenn auch in relativ geringem Umfang, gemessen an den gesamten Transaktionskosten (s. o.).

\subsection{Grenzen der Aussagen}

Die Ergebnisse dieser Arbeit werden insbesondere durch zwei Faktoren eingeschränkt: 1) die Quantifizierung der Transaktionskosten sowie 2) die begrenzte Fallzahl infolge der sehr aufwändigen Erhebungen und der Tatsache, dass viele Unternehmen den Aufwand scheuen, ihre Transaktionskosten in den vier Phasen zu beziffern.

\subsubsection{Datenerhebung}

Die erhoben Zeiten für transaktionskostenrelevante Tätigkeiten der Energieeffizienz-Maßnahmen basieren zum größten Teil auf Schätzwerten der Verantwortlichen. Kein Unternehmen gab an, die zeitlichen Aufwendungen exakt angeben zu können. Grund hierfür sind vor allem die Kostenrechnungssysteme der Unternehmen, in denen der Aufwand für transaktionskostenrelevante Tätigkeiten als Bestandteil der Gemeinkosten angesehen wird, d. h. keine Investitions-scharfen Stundenaufschreibungen erfolgen, und damit im Nachhinein nicht mehr genau nachvollziehbar ist. Die ermittelten Personalkosten sind daher mit Unsicherheit behaftet.

Aber auch allgemein besteht die Problematik, dass selbst innerhalb der Transaktionskostentheorie eine eindeutige Abgrenzung der Transaktionskosten nicht immer möglich ist. Zum Beispiel können Teile der Suchkosten als Fortbildungskosten angesehen werden, oder bei der Entscheidungsphase werden mit der Geschäftsführung mehrere Investitionen zugleich besprochen. Die Quantifizierung der Transaktionskosten hat daher eher die Merkmale subjektiver Einschätzung (vgl. Weber et al. 2001, S. 432).

Weiterhin wurden in der Analyse keine Ressourcen erfasst, die nicht explizit als Kosten ausgewiesen sind bzw. vom Unternehmen nicht als solche erfasst werden. Hierunter fallen beispielsweise Kosten mangelnder Motivation oder auch Opportunitätskosten (Weber et al. 2001, S. 430). Es könnte daher eine Unterbewertung der tatsächlichen Transaktionskosten vorliegen.
Ein weiterer Kritikpunkt ist, dass nur Transaktionskosten zu Energieeffizienz-Investitionen erfasst werden, bei denen eine Umsetzung erfolgte. In Unternehmen fallen aber auch Informationskosten zu Maßnahmen an, bei denen sich anschließend gegen eine Umsetzung entschieden wird. Werden solche Informationskosten nicht erfasst, liegt nach Hein und Blok (1995) eine Unterschätzung der gesamten Informationskosten bei Energieeffizienz-Maßnahmen vor.

\subsubsection{Datenauswertung}

In Abschn. 5 wurden mögliche Einflussfaktoren auf die Transaktionskosten untersucht. Es hier betont, dass diese Arbeit wegen der geringen Fallzahl nicht versucht, kausale Beziehungen zwischen den Variablen zu beschreiben. Die statistischen Analysen zeigen lediglich die Zusammenhänge zwischen den betrachteten Variablen, welche anhand des theoretischen Konzepts der Einflussfaktoren interpretiert werden können.

Die empirisch analysierten Variablen erfassen nur spezielle Zusammenhänge auf Basis von theoretischen Überlegungen. Diese Überlegungen stellen wahrscheinlich nur einen Ausschnitt der Realität dar und dürften daher kaum das gesamte Phänomen der Transaktionskosten erklären können. Es muss vielmehr davon ausgegangen werden, dass noch andere Einflussfaktoren die Transaktionskosten von Energieeffizienz-Investitionen beeinflussen.

Im Zuge der Ermittlung der Transaktionskosten ist auch die Berechnung der Personalkosten mit Unsicherheiten verbunden. Die Bewertung für die zeitlichen Aufwendungen der Mitarbeiter beruht in den meisten Fällen auf einer Annahme des Stundensatzes. Dabei kann der angenommene Wert abhängig vom betrachteten Unternehmen zu hoch oder zu niedrig angesetzt sein.

Abschließend soll noch einmal verdeutlicht werden, dass sich die Aussagen der vorliegenden Arbeit nur auf Unternehmen beziehen, die in einem Energieeffizienz-Netzwerk vertreten sind. Schon aus der Teilnahme an einem solchen Netzwerk lässt sich ableiten, dass in diesen Unternehmen ein Interesse an Energieeffizienzthemen besteht. Zudem werden die Unternehmen im Rahmen von regelmäßigen Netzwerktreffen durch Vorträge, Diskussionen, Erfahrungsaustausch und Betriebsbegehungen über energieeffiziente Technologien und organisatorische Maßnahmen, Preise und Anbieter am Markt informiert, d. h. einer systemtischen Fortbildung ausgesetzt. Dadurch können die Unternehmen ihre Informationskosten senken, was ein großer Teil der 366 befragten Betriebe auch bestätigt. Für Unternehmen außerhalb solcher Energieeffizienz-Netzwerke dürften die Transaktionskosten vermutlich etwas höher ausfallen. 


\section{Schlussbetrachtung und Ausblick}

Gegenstand der vorliegenden Studie ist eine empirische Untersuchung von Transaktionskosten bei EnergieeffizienzInvestitionen von mittelständischen Unternehmen, meist des Verarbeitenden Gewerbes. Über eine Befragung wurden die Transaktionskosten aus Sicht der investierenden Unternehmen erhoben und anschließend analysiert. Als Ergebnis wurden zwei Faktoren identifiziert, die im Zusammenhang mit den relativen Transaktionskosten stehen. Zum einen wurde nachgewiesen, dass eine eindeutige negative Korrelation zwischen der Investitionssumme einer Energieeffizienz-Investition und den relativen Transaktionskosten besteht. Zum anderen korreliert die Energieintensität eines Unternehmens negativ mit den relativen Transaktionskosten. So können vor allem bei niedrigen Investitionssummen und Unternehmen mit einem geringen Jahresenergieverbrauch die Anteile der Transaktionskosten an den jeweiligen Investitionen beachtliche Größenordnungen erreichen. Die These, dass Transaktionskosten als Hemmnis bei Investitionen in Energieeffizienz auftreten können (vgl. Jochem et al. 2010), kann damit bekräftigt werden. Allerdings ist die hemmende Wirkung der Transaktionskosten begrenzt, weil ihr Anteil an der Investitionssumme bei Investitionen über $60.000 €$ unter $20 \%$ fällt und damit die Wirtschaftlichkeit von Energieeffizienz-Investitionen nicht in Frage zu stellen.

Die Ergebnisse sind dabei als erster Ansatz zu verstehen, mögliche Einflussfaktoren der Transaktionskosten bei Energieeffizienz-Investitionen empirisch zu erforschen. Für ein umfassendes Verständnis solcher Einflussfaktoren sind weitere Analysen und ein größeres Sample erforderlich. So macht die vorliegende Studie kaum Aussagen darüber, von welcher Art die Zusammenhänge der untersuchten Variablen sind. Solche Aussagen könnten für Unternehmen und beratende Ingenieure von Interesse sein, ebenso auch für die Konzeption von Förderprogrammen der Europäischen Kommission, des Bundes oder der Länderregierungen für Energieeffizienz-Investitionen bis zu bestimmten Investitionssummen, für Energieeffienz-Netzwerke und für Fortbildungsangebote seitens von Fortbildungsanbietern.

Weitere Untersuchungen sind auch hinsichtlich des Einflusses des Erfahrungsaustauschs in EnergieeffizienzNetzwerken notwendig, der wie eine Fortbildung wirkt. Die vorliegende Untersuchung zeigt, dass vor allem Informations- und Suchkosten vermindert werden. Bei weiteren Analysen empfiehlt sich der Einbezug von Unternehmen, die nicht an einem Energieeffizienz-Netzwerk teilnehmen, als Referenzgruppe.

Schließlich ist auch das Konzept der Transaktionskosten weiter bzgl. der Art der Energieeffizienz-Investition zu verfeinern. Denn bei den zwei Fällen mit den höchsten Transaktionskosten stellte sich heraus, dass es sich jeweils um die Suche nach einer neuen prozesstechnischen Lösung han- delte, die mit hohen Informations- und Suchkosten (z. B. Reisekosten) verbunden waren (und nicht um die gewöhnlichen Transaktionskosten einer konventionellen und vorgegebenen Energieeffizienz-Investition).

\section{Literatur}

Bleyl J, Seefeldt F (2012) Energie-Contracting in der Praxis. Eine Evaluation von 55 Contractingprojekten der öffentlichen Hand aus Kundensicht. Entscheidungskriterien für die Modellauswahl. Fraunhofer IFAM, Bremen

BMU und BMWi (Hrsg) (2010) Energiekonzept für eine umweltschonende, zuverlässige und bezahlbare Energieversorgung. Bundesministerium für Umwelt. Berlin, September 2010

Coase RH (1960) The Problem of Social Cost. J Law Econ 3:1-44

Dann J (2013) Transaktionskosten bei Energieeffizienz-Investitionen in Industrieunternehmen - Ein analytisches Modell zu Abschätzung der Höhe relativer Transaktionskosten. Masterarbeit, IIP Institut für Industriebetriebslehre und Industrieelle Produktion und IREES Institut für Ressourceneffizienz und Energiestrategien, Karlsruhe

Eikmeier B, Seefeldt F, Bleyl J, Arzt C (2009) Contracting im Mietwohnungsbau, Bremer Energieinstitut, Prognos, energetic solution. Endbericht. Studie im Auftrag des BMVBS/BBR. Fraunhofer IFAM, Bremen

Erichsen J (2011) Controlling-Instrumente von A-Z: Die wichtigsten Werkzeuge zur Unternehmenssteuerung, 8. Aufl. Rudolf Haufe, München

Fraunhofer-Institut für System- und Innovationsforschung ISI (2014) Bekanntmachung der Förderung von Energieeffizienz- und Klimaschutz-Netzwerken in Deutschland im Rahmen des Projekts „30-Pilot-Netzwerke“. Internetauftritt des 30 Pilotnetzwerke Projekts. http://www.30pilot-netzwerke.de/nw-de/content/Foerderbedingungen.php. Zugegriffen: 24. Okt 2014

Gebhardt T (2011) Transaktionskosten bei Energieeffizienz-Investitionen - Eine empirische Erhebung bei Unternehmen in Energieeffizienz-Netzwerken. Bachelorarbeit, IIP Institut für Industriebetriebslehre und Industrieelle Produktion und IREES Institut für Ressourceneffizienz und Energiestrategien, Karlsruhe

Göbel E (2002) Neue Institutionenökonomik: Konzeption und betriebswirtschaftliche Anwendungen. Lucius \& Lucius, Stuttgart

Grubb M et al (1993) The costs of limiting fossil-fuel $\mathrm{CO}_{2}$ emissions: a survey and analysis. Ann Rev Energy Environ 18:397-478

Gruber E, Brand M (1991) Promoting energy conservation in small and medium-sized companies. Energy Policy 19:279-287

Hein LG, Blok K (1995) Transaction costs of energy efficiency improvements.The European Council for an Energy Efficient Economy (ECCEEE). [Online] 1995. http://www.eceee.org/conference proceedings/eceee/1995/Panel_2/p2_12/.ZZugegriffen: 15. Nov 2014

HOAI (2013) Verordnung über die Honorare für Architekten- und Ingenieurleistungen. Bundesratsbeschluss vom 7.6.2013; Bundesanzeiger 16.7.2013

Jaffe AB, Stavins RB (1994) The energy-efficiency gap. What does it mean? Energy Policy 22(10):804-810

Jochem E, Mai M, Mielicke U (2014): Ungenutzte fördernde Faktoren von Energieeffizienz-Maßnahmen in Unternehmen - eine wenig beachtete Aktivierung der Motivation. Working Paper No. 4, IREES, Karlsruhe

Jochem E, Mai M, Ott V (2010) Energieeffizienznetzwerke - beschleunigte Emissionsminderungen in der mittelständischen Wirtschaft. Z Energiewirtsch 34:21-28

Koot LW et al (1984) Evaluatie van de werking en effectiviteit van de energietoeslag. CvE-TNO, Apeldoorn 
Köwener D, Schleich J (2000) Reducing barriers to energy efficiency in the German energy service companies sector. Fraunhofer Institut für Systemtechnik und Innovationsforschung, Karlsruhe

Levine MD et al (1994) Energy efficiency, market failures, and government policy. Energy Analysis Program, Lawrence Berkeley Laboratory, Berkeley

Osterheld I (2000) Die Transaktionskostenrechnung als strategieorientiertes Informationssystem: Diskussion eines Operationalisierungsvorschlages am Beispiel eines empirischen Akquisitionsproblems. Dissertation, Universität Jena, s.l.

Ostertag K (2003) No-regret potentials in energy conservation: an analysis of their relevance, size and determinants. Physica-Verlag, Heidelberg

Ostertag K et al (2000) Energiesparen - Klimaschutz, der sich rechnet: Ökonomische Argumente in der Klimapolitik. Physica-Verlag, Heidelberg

Richter R, Furubotn EG (2003) Neue Institutionenökonomik: Eine Einführung und kritische Würdigung. (Übers. Streissler M. 3., überarb. und erw. Aufl). Mohr Siebeck, Tübingen

Schmid C et al (2003) Möglichkeiten, Potenziale, Hemmnisse und Instrumente zur Senkung des Energieverbrauchs branchenübergreifender Techniken in den Bereichen Industrie und Kleinverbrauch.
Fraunhofer-Institut für Systemtechnik und Innovationsforschung Forschungsstelle für Energiewirtschaft, Karlsruhe

Schröter M, Buschak D (2009) Energieeffizienz in der Produktion Wunsch oder Wirklichkeit? Energieeinsparpotentiale und Verbreitungsgrad energieeffizienter Techniken. Fraunhofer-Institut für Innovationsforschung ISI, Karlsruhe

Seefeldt F et al (2007) Potenziale für Energieeinsparung und Energieeffizienz im Lichte aktueller Preisentwicklungen. Prognos, Berlin

VDI (1996) VDI-Richtlinie: VDI 6025. Betriebswirtschaftliche Berechnungen für Investitionsgüter und Anlagen. Beuth, Berlin

Wahl F (2012) Transaktionskosten bei Energieeffizienz-Investitionen in Industrieunternehmen - Eine empirische Untersuchung in Energieeffizienz-Netzwerken Deutschlands. Masterarbeit, IIP Institut für Industriebetriebslehre und Industrieelle Produktion und IREES Institut für Ressourceneffizienz und Energiestrategien, Karlsruhe

Weber J, Weißenberger BE, Löbig M (2001) Operationalisierung der Transaktionskosten. In: Jost P-J (Hrsg) Der Transaktionskostenansatz in der Betriebswirtschaftslehre. Schäffer-Poeschel, Stuttgart, S $417-447$

Williamson OE (1990) Die ökonomischen Institutionen des Kapitalismus: Unternehmen, Märkte, Kooperationen. (Übers. Streissler M). Mohr, Tübingen 